\title{
ATLAS/CMS Upgrades
}

\section{Yasuyuki Horii*}

Nagoya University

E-mail: vhoriiehepl.phys.nagova-u.ac.ip

Precision studies of the Standard Model (SM) and searches of the physics beyond the SM are ongoing at the ATLAS and CMS experiments at the Large Hadron Collider (LHC). A luminosity upgrade of LHC is planned, and the collider after the upgrade is called High-Luminosity LHC (HL-LHC). The integrated luminosity of $300 \mathrm{fb}^{-1}$ and $3000 \mathrm{fb}^{-1}$ are foreseen for LHC and HLLHC, respectively. The increased luminosity provides a significant challenge for the experiments, including higher radiation dose, higher particle rate, higher pileup, and higher event rate. The upgrades are essential for the ATLAS and CMS experiments in various aspects, and the planning is underway. With the high statistics data, Higgs boson couplings to fermions are expected to be measured with a precision of the order of percent for various flavors, which provides precision tests of the Yukawa terms of the Lagrangian. A measurement of the $B_{s, d}$ meson and top quark decays through flavor changing neutral current processes provides the probes for the physics beyond the SM.

Flavor Physics and CP Violation,

6-9 June 2016

Caltech, Pasadena CA, USA

* On behalf of the ATLAS and CMS Collaborations. 


\section{Introduction}

Precision studies of the Standard Model (SM) and searches of the physics beyond the SM are ongoing at the ATLAS [四] and CMS [2] experiments at the Large Hadron Collider (LHC) [3]. A luminosity upgrade of LHC is planned, which provides a significant challenge for the experiments. In this report, the plans of the ATLAS and CMS upgrade programs are discussed. Physics prospects for selected topics, including Higgs boson coupling measurements, $B_{s, d} \rightarrow \mu \mu$ decays, and top quark decays through a flavor changing neutral current (FCNC), are also shown.

\section{LHC and HL-LHC Plan}

ATLAS and CMS are currently taking data at the LHC at a centre-of-mass energy of $13 \mathrm{TeV}$ of the colliding protons. It is foreseen to increase the centre-of-mass energy up to $14 \mathrm{TeV}$ in the coming years. An integrated luminosity of $300 \mathrm{fb}^{-1}$ is expected by the end of 2023 . A luminosity upgrade of LHC is planned from 2024 to 2026 [䧃]. The collider after the upgrade is called HighLuminosity LHC (HL-LHC). The expected peak luminosity of HL-LHC is $5-7 \times 10^{34} \mathrm{~cm}^{-2} \mathrm{~s}^{-1}$, where the estimated average pileup is $140-200$ events. An integrated luminosity of $3000 \mathrm{fb}^{-1}$ is expected after 10 years of operation of HL-LHC. Figure $\square$ summarises the plan for LHC and HL-LHC.

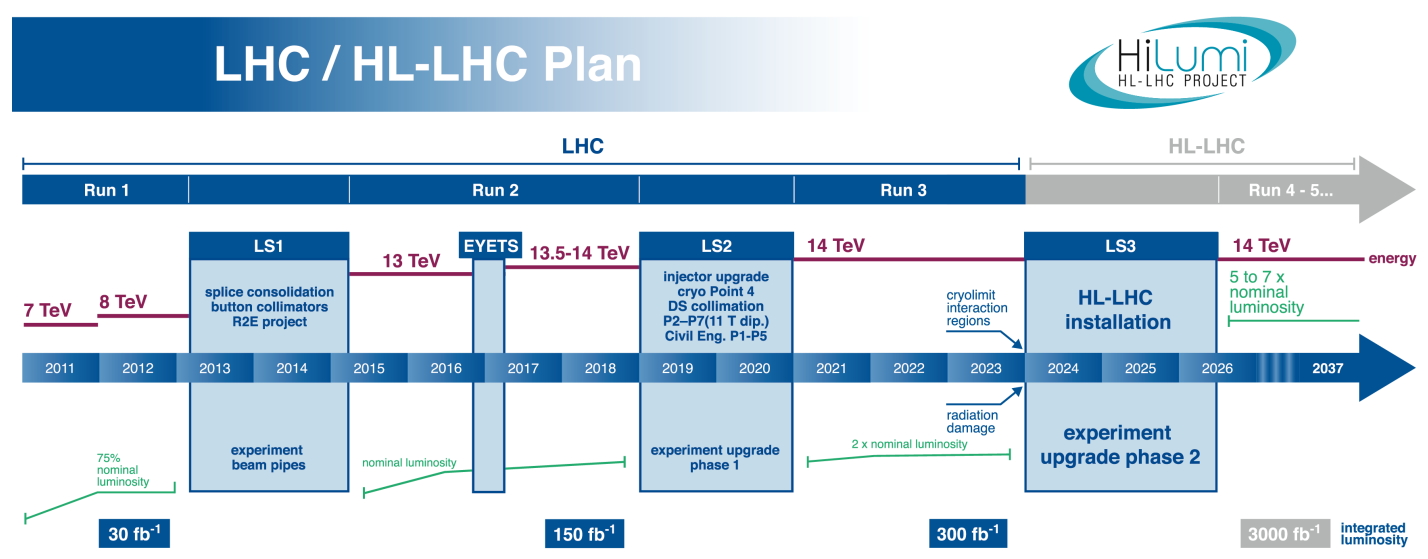

Figure 1: Overview of the LHC and HL-LHC plans [䧃].

\section{ATLAS and CMS Upgrade Programs}

An increased luminosity provides a significant challenge for the ATLAS and CMS experiments. We expect higher radiation dose, higher particle rate, higher pileup, and higher event rate. The upgrades of the ATLAS and CMS experiments are essential to exploit the full potential of LHC and HL-LHC. Some parts of the detectors and of the electronics have to be replaced. The latter is necessary for the overall modification on the trigger and readout scheme. The upgrades are planned in two phases: Phase 1 in 2019-2020 and Phase 2 in 2024-2026. The proposals of the Phase 2 upgrades are described in Ref. [15, 6]. 


\subsection{Inner Tracker Upgrades}

The inner trackers will be in an extreme environment at HL-LHC. In fact in the region close to the beam pipe, the expected $1 \mathrm{MeV}$ neutron equivalent fluence is up to about $2 \times 10^{16} \mathrm{~cm}^{-2}$. The estimated ionisation dose for 10 years of operation of HL-LHC is up to about $10 \mathrm{MGy}$. The simulated particle rate is up to about $2 \times 10^{9} \mathrm{~cm}^{-2} \mathrm{~s}^{-1}$.

The current inner tracker of the ATLAS experiment consists of pixel and strip semiconductor detectors and a straw-tube transition radiation tracker [四]. An entire replacement of the inner tracker is planned at the Phase 2 upgrade. An all-silicon inner tracker is proposed as the new inner tracker. The current inner tracker of the CMS experiment consists of pixel and strip semiconductor detectors. A pixel detector replacement is planned from the end of 2016 to the beginning of 2017 [ $[$ ] . An entire replacement of the inner tracker is planned for the Phase 2 upgrade.

The concepts of the Phase 2 upgrades of the inner tracker are similar for the ATLAS and CMS experiments. The new inner tracker is designed to have higher radiation tolerance, increased granularity, and reduced material. An extension of the coverage in the forward region is proposed. Figure $\square$ (a) shows a proposed layout of the new inner tracker of the ATLAS experiment. The layout is provided with a large number of layers to suppress the fake tracks. The pixel thickness in the inner layers is possibly $150 \mu \mathrm{m}$ to increase the radiation tolerance. The pixel size is possibly $50 \mu \mathrm{m} \times 50 \mu \mathrm{m}$, which provides an increased granularity and robustness against a higher pileup. Figure $\square$ (b) shows the estimated radiation length for the inner tracker of the CMS experiment. The radiation length is reduced thanks to the changes in the control electronics and the conductors bringing the current to the front-end electronics. The semiconductor modules are the main contributor to the total material of the detector in the new inner tracker.

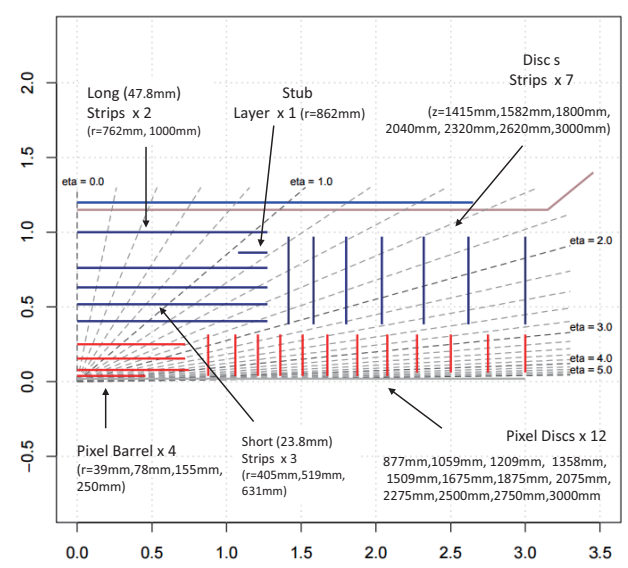

(a) Proposed layout of the new inner tracker at ATLAS. The coverage of the pixel detector (red) and the strip detector (blue) are shown. Horizontal and vertical lines represent barrel and endcap layers, respectively. The blue line outside the tracker volume indicates the coil of the solenoid magnet.

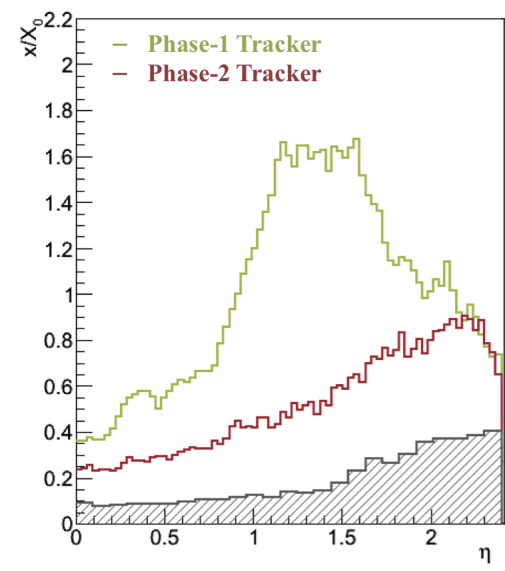

(b) Radiation length as a function of the pseudorapidity for the material inside the tracking volume for Phase 1 (green) and Phase 2 (red) trackers at CMS. The expected contribution of the Phase 1 pixel detector (black hashed) is provisionally used also for the Phase 2 tracker.

Figure 2: Proposed layout of the inner tracker at ATLAS (a) [四] and the radiation length for CMS (b) [6]. 


\subsection{Calorimeter Upgrades}

At the CMS experiment, the endcap calorimeter will be replaced at the Phase 2 upgrade to increase the longevity and the performances. The high-energy charged hadron fluence in the region at the pseudorapidity of 2.6 is expected to be $2 \times 10^{14} \mathrm{~cm}^{-2}$. Defects are expected in lead tungstate scintillating crystal of the electromagnetic calorimeter, which result in the light transmission loss. Response degradation is also expected for the hadron calorimeter. A high-granularity sampling calorimeter is proposed with a tungsten-silicon electromagnetic part followed by brass-silicon and brass-scintillator hadronic parts. Figure [ ] shows a drawing of the new endcap calorimeter. Recent advances in the silicon detectors in terms of cost per unit area and radiation tolerance are exploited. The new calorimeter is designed to be less sensitive to pileup.

The detectors of the ATLAS calorimeter system, including the liquid argon electromagnetic calorimeters, the scintillating tile hadronic barrel detectors, and the hadronic endcap calorimeter, are expected to maintain their performance under HL-LHC conditions and therefore do not need replacement. On the other hand, the performance of the liquid argon forward calorimeters could be degraded by the high energy and particle density at HL-LHC. The replacement of the liquid argon forward calorimeters with high-granularity calorimeters is under discussion. The addition of timing detectors with an intrinsic time resolution of a few tens of picoseconds is also under discussion. Precision timing would allow the association of energy clusters in the calorimeter to a small area of the luminous region around the primary vertex to mitigate the pileup effects.

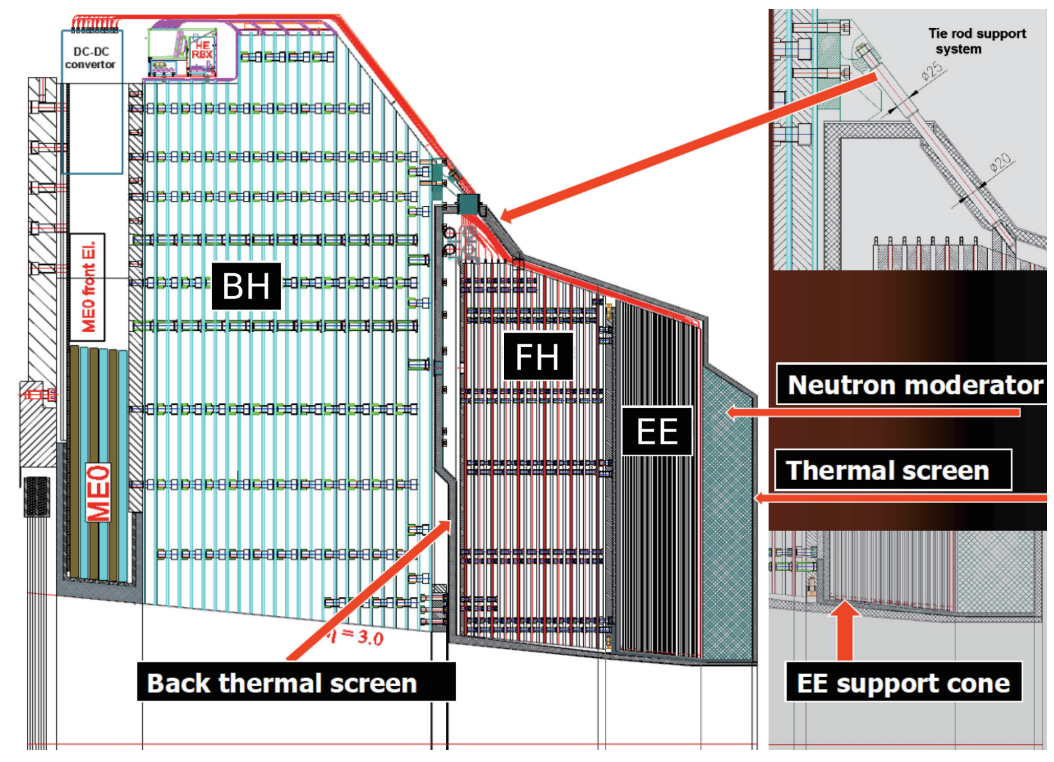

Figure 3: Drawing of the overall structure of the new endcap calorimeter of CMS [四], which is a highgranularity sampling calorimeter with a tungsten-silicon electromagnetic part (EE) followed by brass-silicon (FH) and brass-scintillator (BH) hadronic parts. 


\subsection{Muon Spectrometer Upgrades}

At the ATLAS experiment, efficiency loss and position resolution degradation are expected for the drift tube chambers in the small wheels. The small wheels are located in the endcap inner layers of the muon system, as indicated in Figure 7 (a). The small wheels will be replaced by new ones which consist of small-strip thin-gap chambers (sTGC) and micro-mesh gaseous detectors (Micromegas) [ []]. Figure $\$$ (b) shows the structure of the new small wheel. Both sTGC and Micromegas are planned to be used for precision tracking and trigger. Position resolution per layer for the precision tracking is expected to be about $100 \mu \mathrm{m}$. Track segments are reconstructed at the first-level trigger, which play an important role to suppress the fake first-level muon triggers generated by low momentum particles not originating from the interaction region. The angular resolution for the segment is expected to be $1 \mathrm{mrad}$

At the CMS experiment, it is proposed to install additional chambers that make use of new detector technologies, including gas electron multiplier chambers and low-resistivity resistive plate chambers with improved timing resolution. The additional chambers improve the momentum resolution for the standalone muon trigger and also increase the coverage for muon detection to a pseudorapidity of about 3 .

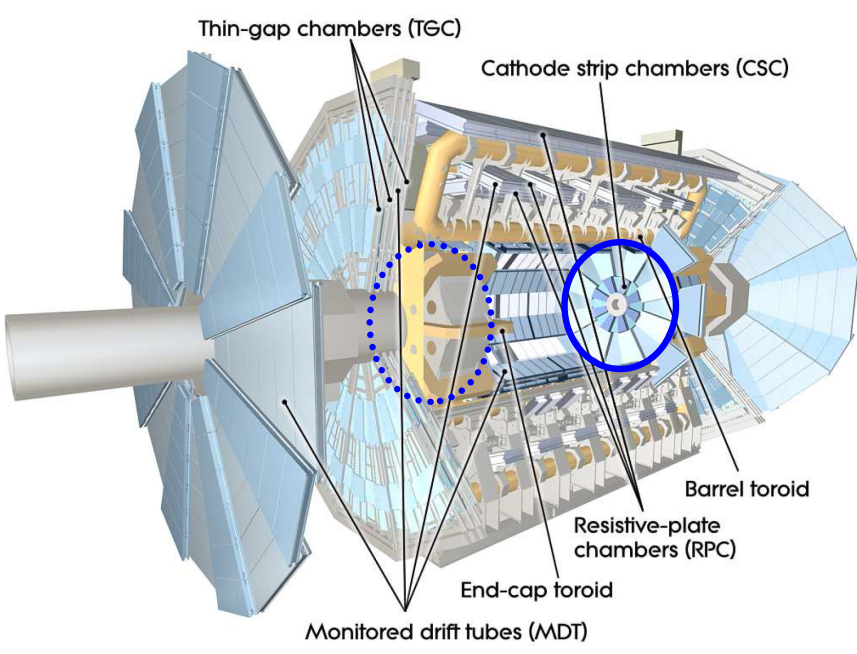

(a) Cut-away view of the ATLAS muon system. The locations of the small wheels are indicated by the blue circles.

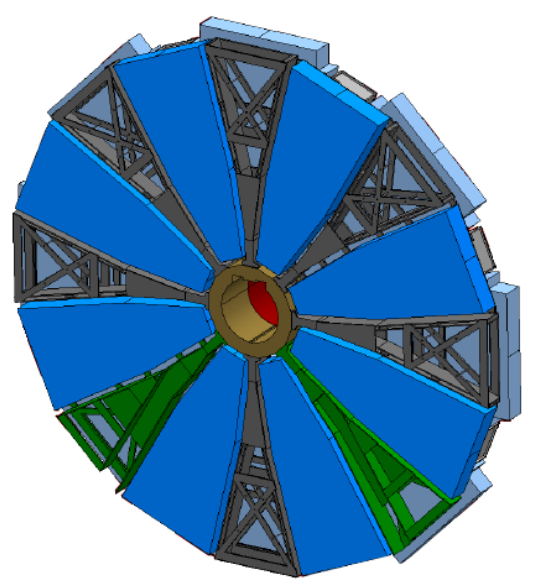

(b) Structure of the new small wheel of the ATLAS muon system.

Figure 4: Locations of the small wheels (a) and the structure of the new small wheel (b) at ATLAS [ [ ]].

\subsection{Trigger Upgrades}

With the increased luminosity, more interesting events are produced but also more background events are produced. Without changes in the trigger and readout scheme, the trigger rates would exceed the limits of the trigger and readout system. Simply increasing the threshold to reduce the trigger rates results in the acceptance loss for physics processes. The choice of the ATLAS and CMS experiments is to increase the allowed trigger rates and latency. The trigger rate and the latency for the first-level trigger described in Ref. [5], 囵] are $1 \mathrm{MHz}(750 \mathrm{kHz})$ and $10 \mu \mathrm{s}$ 
(12.5 $\mu \mathrm{s})$ for the ATLAS (CMS) experiment. The replacement of the electronics is essential for all subsystems with higher bandwidth of the data transfer and larger buffer for storing data before the trigger decision. The following discussions focus on the first-level trigger. Substantial upgrades are also planned for the higher-level triggers.

At the CMS experiment, the implementation of the tracker-based trigger in the first-level trigger is planned for the Phase 2 upgrade. The benefits include improved $p_{\mathrm{T}}$ determination and better identification of charged leptons. Studies are ongoing to use associative memories and fieldprogrammable gate arrays. Figure 5 (a) shows the efficiency for the first-level muon trigger as a function of the muon transverse momentum. With the tracker-based trigger, the transverse momentum resolution is improved and the first-level muon trigger rate is reduced. Figure इ (b) shows the trigger rate for the first-level electron trigger depending on transverse energy threshold. With the tracker-based trigger, the matching between the clusters in the calorimeter and the tracks is obtained with different thresholds for electrons and photons. Significant rate reduction is expected for the first-level electron trigger.

At the ATLAS experiment, upgrades are planned for the first-level calorimeter and muon trigger systems. The current first-level calorimeter trigger relies on the signal collected in cells of pseudorapidity and azimuthal angle of $0.1 \times 0.1$. A high granularity information will be provided after the Phase 1 upgrade [Q]. Trigger rate reduction is expected for electrons, photons, and other objects. The product of the acceptance and the efficiency for muons in the current first-level muon trigger in the barrel is about 70\% due to a limited coverage of the detectors. At the Phase 2 upgrade, additional resistive plate chambers will be installed in the barrel inner layer. The product of the acceptance and the efficiency could be improved up to about $95 \%$.

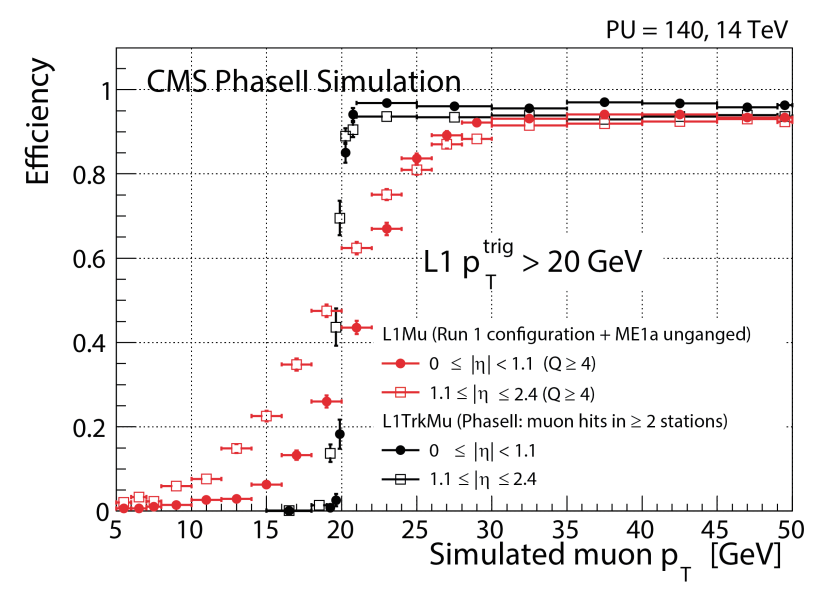

(a) Efficiency for a single muon trigger with $20 \mathrm{GeV}$ threshold as a function of the generated transverse momentum of the muon. The distributions are shown for muon candidates reconstructed from muon spectrometer (red symbols) and muon candidates obtained with track matching (black symbols).

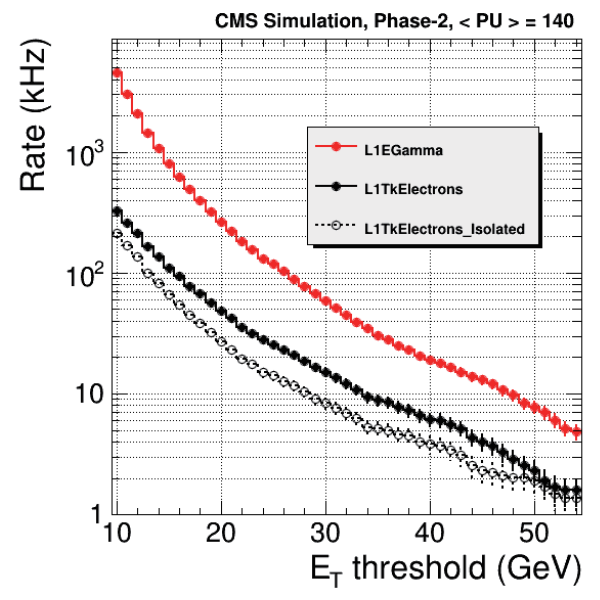

(b) Rate for a single electron trigger for electron and photon candidates obtained from calorimeter (red dots), electron candidates obtained with track matching (black dots), and ones obtained with tracker-based isolation (black open dots).

Figure 5: Expected performance of the first-level tracker-based trigger for the muons (a) and the electrons (b) at CMS [司]. 


\section{Physics Prospects}

The increased luminosity provides an improved precision of the SM measurements and an extended sensitivity of the searches of the physics beyond the SM. Physics prospects will be discussed for the Higgs boson coupling measurements and the indirect searches of the physics beyond the SM with the processes including $B_{s, d}$ mesons and top quarks.

\subsection{Higgs Physics}

Higgs boson production in association with top-quark pair $(t \bar{t} H)$ provides a direct measurement of the coupling between the Higgs boson and the top quarks. The coupling between the Higgs boson and the top quarks can also be accessed from the $g g \rightarrow H$ production and the $H \rightarrow \gamma \gamma$ decay, where the top quark contributes in the loop diagram. Figure 6 (a) shows a simulation result of the ATLAS experiment for the $t \bar{t} H$ production followed by the $H \rightarrow \gamma \gamma$ decay at an integrated luminosity of $3000 \mathrm{fb}^{-1}$ [ए]]. The expected significance at $3000 \mathrm{fb}^{-1}$ is 8.2 standard deviations.

The Higgs boson decay $H \rightarrow b \bar{b}$ is sensitive to the coupling between the Higgs boson and the bottom quarks. The most sensitive production mode is $V H$, where $V$ indicates a $Z$ or $W$ boson. By employing leptonic decays of the $V$ boson, the background contribution is significantly suppressed. Figure 6 (b) shows a simulation result of the ATLAS experiment for the $Z H$ production followed by the $H \rightarrow b \bar{b}$ decay at an integrated luminosity of $3000 \mathrm{fb}^{-1}$ [प]]. The expected significance with $3000 \mathrm{fb}^{-1}\left(300 \mathrm{fb}^{-1}\right)$ is 8.8 (3.9) standard deviations.

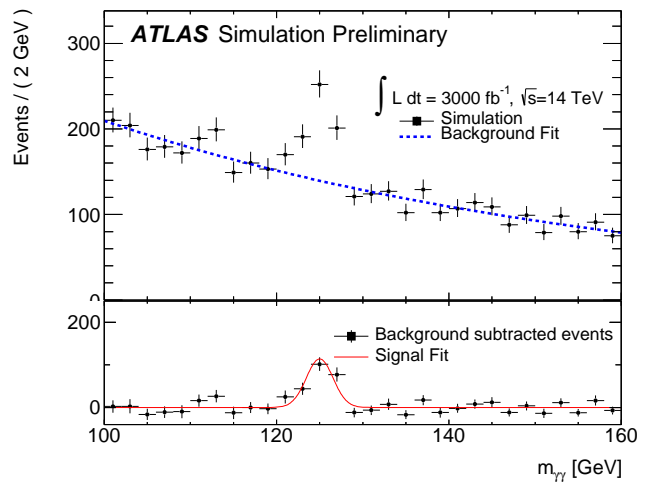

(a) A simulation result of ATLAS for the $t \bar{t} H$ production followed by the $H \rightarrow \gamma \gamma$ decay at $3000 \mathrm{fb}^{-1}$. Distributions of the invariant mass of $\gamma \gamma$ are shown.

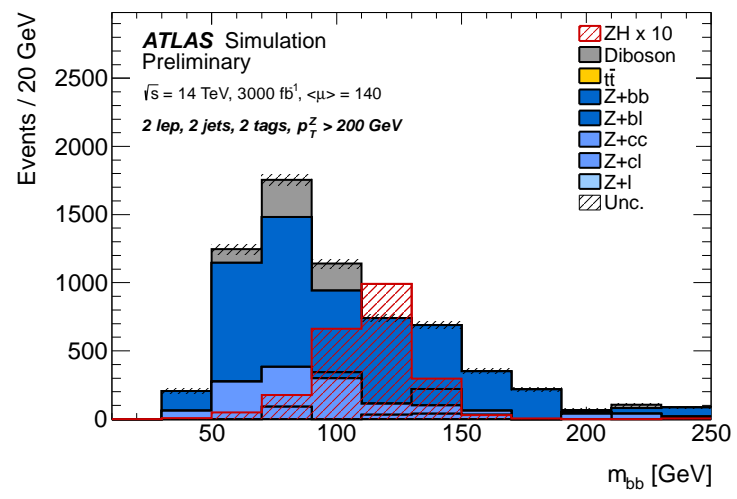

(b) A simulation result of ATLAS for the $\mathrm{ZH}$ production followed by the $H \rightarrow b \bar{b}$ decay at $3000 \mathrm{fb}^{-1}$. Distributions of the invariant mass of $b \bar{b}$ are shown.

Figure 6: Prospects for the $t \bar{t} H$ production (a) [ए人] and the $H \rightarrow b \bar{b}$ decay (b) [ए]].

The Higgs boson decay $H \rightarrow \mu \mu$ is sensitive to the coupling between the Higgs boson and the muons. Figure $\square$ (a) shows the invariant mass distributions for two muons simulated for the CMS experiment. With the inner tracker upgrade at the Phase 2 upgrade, the invariant mass resolution is expected to be improved by $40 \%$. Figure $\square$ (b) shows a simulation result of the ATLAS experiment for the $H \rightarrow \mu \mu$ decay at an integrated luminosity of $3000 \mathrm{fb}^{-1}$ [प]]. The expected significance at $3000 \mathrm{fb}^{-1}$ is 7.0 standard deviations. 


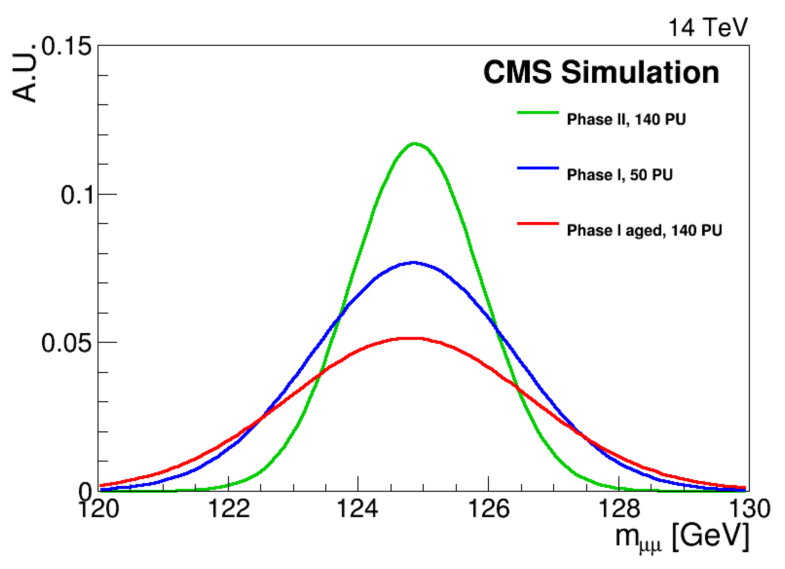

(a) Normalised distributions of the invariant mass of $\mu \mu$ simulated with Phase 1 and Phase 2 CMS detectors. Two conditions are assumed for Phase 1, pileup of 50 without radiation damage and pileup of 140 with radiation damage for $1000 \mathrm{fb}^{-1}$.

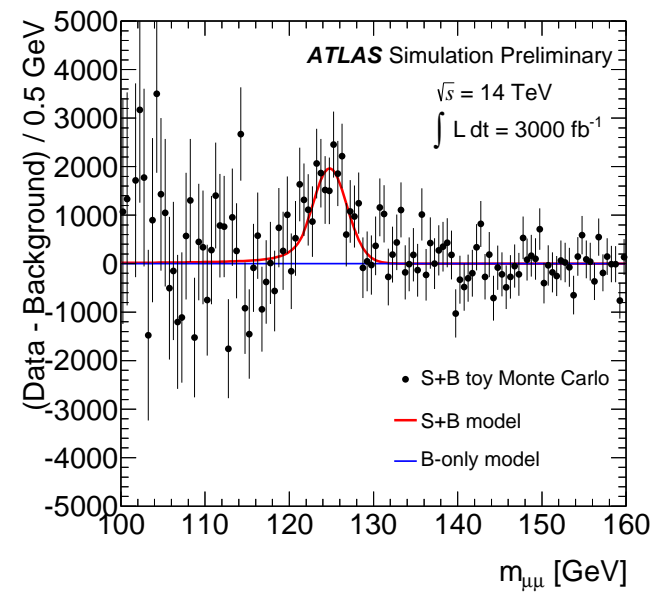

(b) A simulation result of ATLAS for the $H \rightarrow \mu \mu$ decay at $3000 \mathrm{fb}^{-1}$. Background subtracted distribution of the invariant mass of $\mu \mu$ is shown.

Figure 7: Comparison of the invariant mass distributions for the $H \rightarrow \mu \mu$ signal (a) [目] and a simulated background subtracted distribution of the invariant mass for the $H \rightarrow \mu \mu$ analysis (b) [D]].

Figure 8 (a) summarises the expectation for the Higgs boson coupling measurements at the ATLAS experiment [ए33]. The precision of the ratios of the coupling scale factors are shown for $300 \mathrm{fb}^{-1}$ and $3000 \mathrm{fb}^{-1}$. The estimations are based on a fit with a fully generic parametrisation, where neither the assumption on the total width nor the assumption on new particle contribution through loop is included. Even with such a generic fit, for various coupling scale factor ratios, the precision of the order of percent is expected at $3000 \mathrm{fb}^{-1}$. Figure $\mathbb{B}$ (b) shows a simulation result of the CMS experiment for $3000 \mathrm{fb}^{-1}$ for the relation between the coupling scale factor and the mass of the particle coupled to the Higgs boson. Precision tests of the Yukawa terms in the Lagrangian are possible for various flavors: top quark, bottom quark, tau lepton, and muon. The tests include up-type and down-type, quark and lepton, and third and second generations.

\subsection{B Physics}

Rare decays that are well-predicted in the SM provide the opportunities of the indirect searches of the physics beyond the SM. The $B_{s} \rightarrow \mu \mu$ and $B_{d} \rightarrow \mu \mu$ decays only proceed through the processes including FCNC and are highly suppressed in the SM. The SM predicts the branching ratios of the $B_{s} \rightarrow \mu \mu$ and $B_{d} \rightarrow \mu \mu$ decays to be $(3.65 \pm 0.23) \times 10^{-9}$ and $(1.06 \pm 0.09) \times 10^{-10}$ [[4]], respectively. Some of the physics scenarios beyond the SM may boost the decay rates, and the $B_{s} / B_{d}$ ratio provides a stringent test of different scenarios [ㄷ]]. Figure $\mathbf{Q}$ (a) shows a simulation result of the CMS experiment for the $B_{s, d} \rightarrow \mu \mu$ decays at $3000 \mathrm{fb}^{-1}$ assuming the SM branching ratios. The expected precision for $B_{s} \rightarrow \mu \mu$ and $B_{d} \rightarrow \mu \mu$ branching ratios are $11 \%$ and $18 \%$, respectively. 
ATLAS Simulation Preliminary $\sqrt{\mathrm{s}}=14 \mathrm{TeV}: \int \mathrm{Ldt}=300 \mathrm{fb}^{-1} ; \int \mathrm{Ldt}=3000 \mathrm{fb}^{-1}$

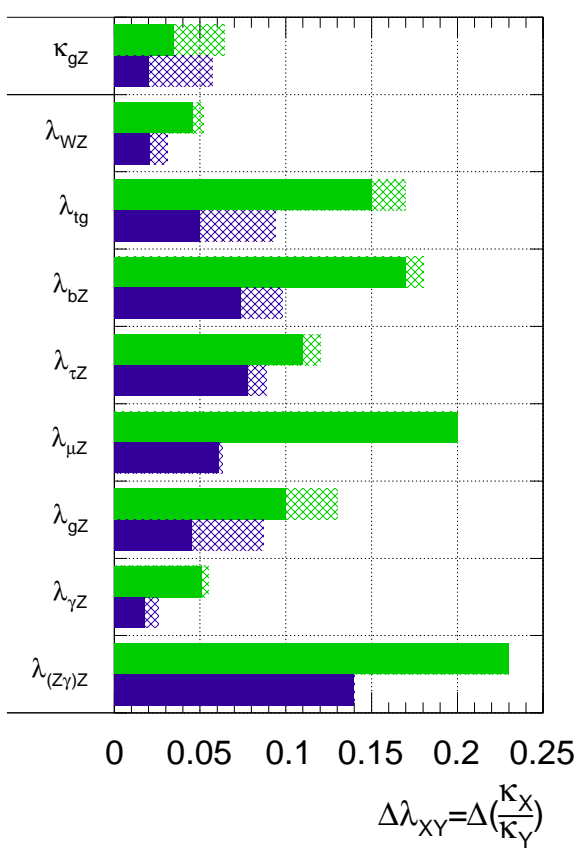

(a) Relative uncertainty expected at ATLAS for the coupling scale factor ratios $\lambda_{X Y}$ in a generic fit without assumptions. The hashed areas indicate the increase of the estimated error due to current theory systematic uncertainties.

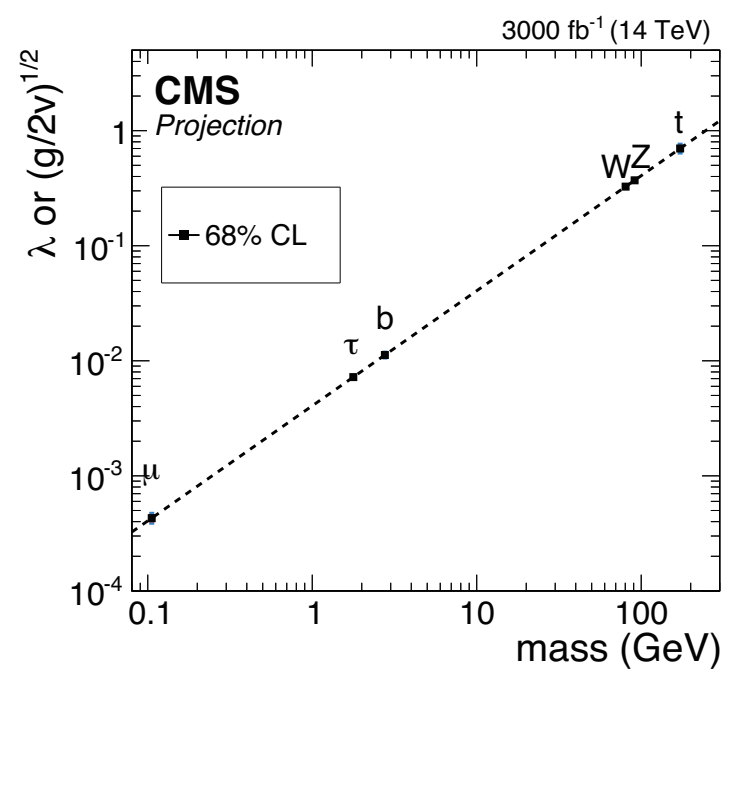

(b) Precision on the Higgs boson couplings as a function of fermion or boson masses at CMS projected for $3000 \mathrm{fb}^{-1}$. The couplings of fermions and bosons are parametrised to be $\kappa_{f} \frac{m_{f}}{v}(\lambda)$ and $\sqrt{\kappa_{V}} \frac{m_{V}}{v}\left((g / 2 v)^{1 / 2}\right)$, respectively, to preserve a linear mass dependence.

Figure 8: Prospects for the Higgs boson coupling measurements (a) [ए3] and the relation between the coupling and the particle mass (b) [6].

$C P$ asymmetry in the $B_{s} \rightarrow J / \psi \phi$ decay could arise from the interference between the direct decay and the decay through $B_{s}^{0}-\bar{B}_{s}^{0}$ mixing. Physics beyond the SM could show up in the mixing. Phase difference between the interfering amplitudes $\phi_{s}$ is extracted from the proper decay time $\tau=\frac{L_{x y} M_{B}}{c p_{\mathrm{T}}}$, where $L_{x y}$ is the flight length of the $B_{s}$ meson in the transverse plane, $p_{\mathrm{T}}$ is the momentum of the $B_{s}$ meson in the transverse plane, and $M_{B}$ is the mass of the $B_{s}$ meson. Figure $Q$ (b) shows the proper decay time resolution simulated at the ATLAS experiment [16]. The additional pixel layer, which was installed in 2014, improves the resolution by $30 \%$. The statistical errors on $\phi_{s}$ estimated for $250 \mathrm{fb}^{-1}$ and $3000 \mathrm{fb}^{-1}$ are $0.064 \mathrm{rad}$ and $0.022 \mathrm{rad}$, respectively. They are compared with the SM global fit result provided by the CKMfitter group of $\phi_{s}=-0.0365_{-0.0012}^{+0.0013} \mathrm{rad}$ [एँ]].

\subsection{FCNC in Top Decays}

Top quark decays $t \rightarrow q \gamma, t \rightarrow q Z$, and $t \rightarrow q H$, where $q$ indicates a charm or up quark, proceed through FCNC processes and are highly suppressed in the SM. Some of the physics scenarios beyond the SM may enhance the branching ratios up to the order of $10^{-3}$ [ए8]. Figure [0] (a) shows the already available and the foreseen exclusion regions for the branching ratios of the $t \rightarrow q \gamma$ and 


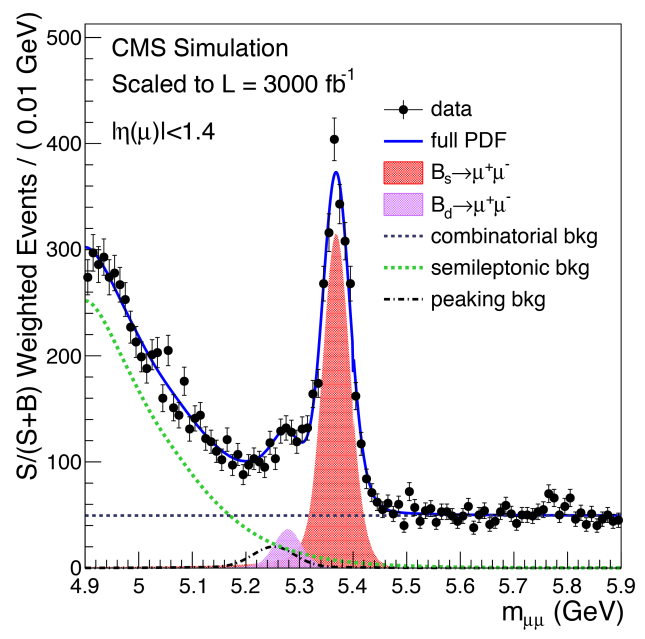

(a) A simulation result of CMS for the $B_{s, d} \rightarrow \mu \mu$ decays at $3000 \mathrm{fb}^{-1}$. Distribution of the invariant mass of $\mu \mu$ is shown. Two peaks corresponding to $B_{s}$ and $B_{d}$ are resolved by the improved tracking.

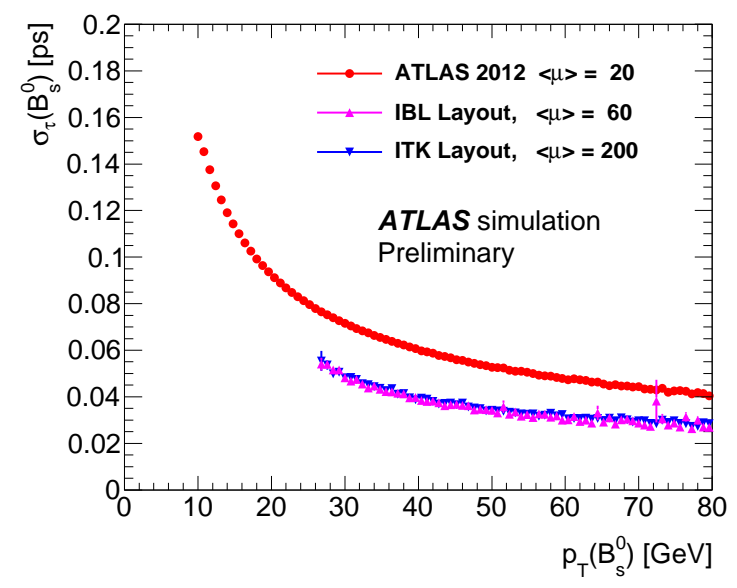

(b) Proper decay time resolution for the $B_{S} \rightarrow J / \psi \phi$ decay simulated at ATLAS. Three conditions are considered: the detector in 2012 with pileup of 20, the Phase 1 detector with pileup of 60 , and the Phase 2 detector with pileup of 200.

Figure 9: Prospects for the $B_{s, d} \rightarrow \mu \mu$ decays (a) [6] and the $B_{s} \rightarrow J / \psi \phi$ decay (b) [ए]].

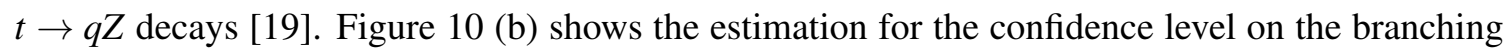
ratio of the $t \rightarrow c H$ decay [ [20]. The sensitivities are expected to reach the levels of $10^{-4}$ to $10^{-5}$ at HL-LHC, providing stringent tests of the physics beyond the SM.

\section{Summary}

Precision studies of the SM and searches of the physics beyond the SM are ongoing at the ATLAS and CMS experiments. A luminosity upgrade of LHC is planned, and the collider after the upgrade is called HL-LHC. Integrated luminosities of $300 \mathrm{fb}^{-1}$ and $3000 \mathrm{fb}^{-1}$ are foreseen for LHC and HL-LHC, respectively. The increased luminosity provides a significant challenge for the experiments, which have to face higher radiation doses, higher particle rates, a higher pileup, and a higher event rate. The detector upgrades are essential for the ATLAS and CMS experiments, and their planning is underway. With the high statistics data, the observation is expected of the $t \bar{t} H$ production, the $H \rightarrow b \bar{b}$ decay, and the $H \rightarrow \mu \mu$ decay assuming production and decay rates of the SM. Higgs boson couplings to fermions are expected to be measured with a precision of the order of percent for various flavors, which provides precision tests of the Yukawa terms of the Lagrangian. The ratio between the $B_{s} \rightarrow \mu \mu$ and $B_{d} \rightarrow \mu \mu$ decay rates is sensitive to the physics beyond the SM. Top quark decays through FCNC processes also provide the probes for the physics beyond the SM. The sensitivity of the direct searches is extended by the increased integrated luminosity. 


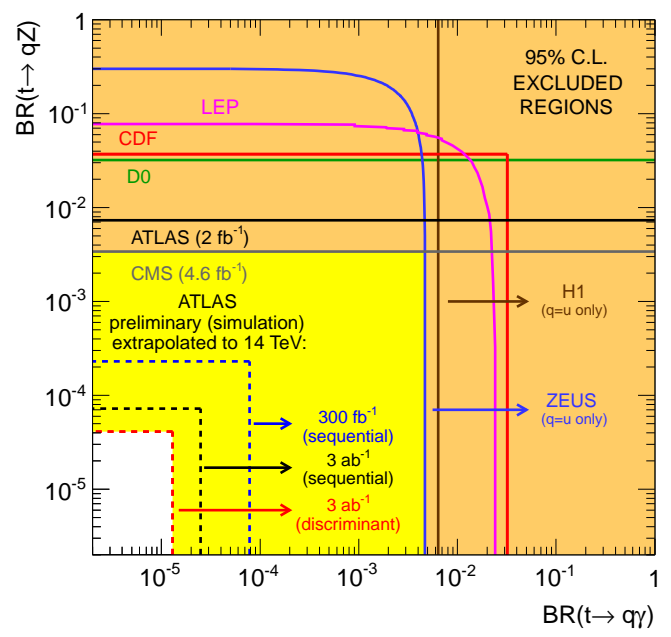

(a) The present observed limits at $95 \%$ confidence level (solid lines) and the expected sensitivities at ATLAS for $300 \mathrm{fb}^{-1}$ and $3000 \mathrm{fb}^{-1}$ (dashed lines) for the branching ratios of the $t \rightarrow q \gamma$ and $t \rightarrow q Z$ decays.

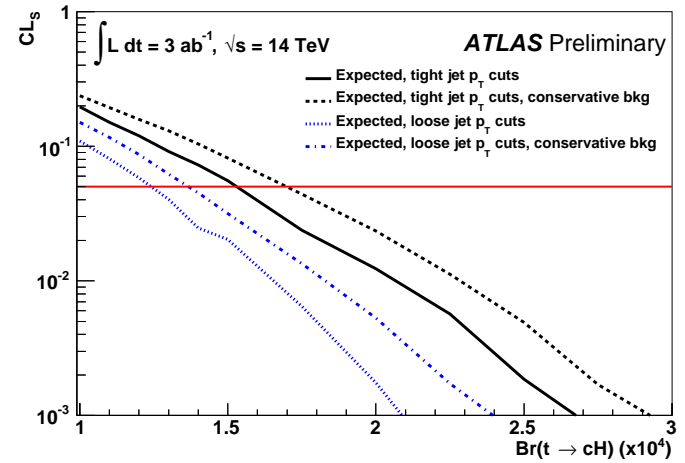

(b) Evolution of the signal confidence level as a function of the branching ratio of the $t \rightarrow c H$ decay simulated at ATLAS for $3000 \mathrm{fb}^{-1}$. Different curves are based on different assumptions on the background and the jet selection.

Figure 10: Prospects for the measurement of the branching ratios of the $t \rightarrow q \gamma$ and $t \rightarrow q Z$ decays (a) [एव] and the expected sensitivity for the branching ratio of the $t \rightarrow c H$ decay (b) [एव]].

\section{References}

[1] ATLAS Collaboration, The ATLAS Experiment at the CERN Large Hadron Collider, JINST 3, S08003 (2008), http://iopscience.iop.org/article/10.1088/1748-0221/3/08/S08003.

[2] CMS Collaboration, The CMS Experiment at the CERN LHC, JINST 3, S08004 (2008), http://iopscience.iop.org/article/10.1088/1748-0221/3/08/S08004.

[3] L. Evans and P. Bryant, LHC Machine, JINST 3, S08001 (2008), http://iopscience.iop.org/article/10.1088/1748-0221/3/08/S08001.

[4] B. Alonso et al., HiLumi LHC Technical Design Report, CERN-ACC-2015-0140 (2015), https://cds.cern.ch/record/2069130.

[5] ATLAS Collaboration, ATLAS Phase-II Upgrade Scoping Document, CERN-LHCC-2015-020; LHCC-G-166 (2015), https://cds.cern.ch/record/2055248.

[6] CMS Collaboration, Technical Proposal for the Phase-II Upgrade of the Compact Muon Solenoid, CERN-LHCC-2015-010; LHCC-P-008 (2015), https://cds.cern.ch/record/2020886.

[7] CMS Collaboration, CMS Technical Design Report for the Pixel Detector Upgrade, CERN-LHCC-2012-016; CMS-TDR-11 (2012), https://cds.cern.ch/record/1481838.

[8] ATLAS Collaboration, New Small Wheel Technical Design Report, CERN-LHCC-2013-006; ATLAS-TDR-020 (2013), https://cds.cern.ch/record/1552862.

[9] ATLAS Collaboration, Technical Design Report for the Phase-I Upgrade of the ATLAS TDAQ System, CERN-LHCC-2013-018; ATLAS-TDR-023 (2013), https://cds.cern.ch/record/1602235. 
[10] ATLAS Collaboration, HL-LHC Projections for Signal and Background Yield Measurements of the $H \rightarrow \gamma \gamma$ when the Higgs Boson is Produced in Association with $t$ Quarks, $W$ or $Z$ Bosons, ATL-PHYS-PUB-2014-012 (2014), https://cds.cern.ch/record/1741011.

[11] ATLAS Collaboration, A Study of Standard Model Higgs Boson Production in the Decay Mode $H \rightarrow b \bar{b}$ in Association with a $W$ or $Z$ Boson for High Luminosity LHC Running, ATL-PHYS-PUB-2014-011 (2014), https://cds.cern.ch/record/1740962.

[12] ATLAS Collaboration, Projections for Measurements of Higgs Boson Cross Sections, Branching Ratios and Coupling Parameters with the ATLAS Detector at a HL-LHC, ATL-PHYS-PUB-2013-014 (2013), https://cds.cern.ch/record/1611186.

[13] ATLAS Collaboration, Projections for Measurements of Higgs Boson Signal Strengths and Coupling Parameters with the ATLAS Detector at a HL-LHC, ATL-PHYS-PUB-2014-016 (2014), http://cds.cern.ch/record/1956710.

[14] C. Bobeth et al., $B_{s, d} \rightarrow \ell^{+} \ell^{-}$in the Standard Model with Reduced Theoretical Uncertainty, Phys. Rev. Lett. 112, 101801 (2014).

[15] D. M. Straub, New Physics Correlations in Rare Decays, arXiv:1012.3893 (2011).

[16] ATLAS Collaboration, ATLAS B-Physics Studies at Increased LHC Luminosity, Potential for $C P$-Violation Measurement in the $B_{s}^{0} \rightarrow J / \psi \phi$ Decay, ATL-PHYS-PUB-2013-010 (2013), http://cds.cern.ch/record/1604429.

[17] J. Charles et al. (CKMfitter Group), Current Status of the Standard Model CKM Fit and Constraints on $\Delta F=2$ New Physics, Phys. Rev. D 91, 073007 (2015).

[18] J. A. Aguilar-Saavedra, Top Flavour-Changing Neutral Interactions: Theoretical Expectations and Experimental Detection, Acta Phys. Pol. B 35, 2695-2710 (2004).

[19] ATLAS Collaboration, Physics at a High-Luminosity LHC with ATLAS, ATL-PHYS-PUB-2013-007 (2013), http://cds.cern.ch/record/1564937.

[20] ATLAS Collaboration, Sensitivity of ATLAS at HL-LHC to Flavour Changing Neutral Currents in Top Quark Decays $t \rightarrow c H$, with $H \rightarrow \gamma \gamma$, ATL-PHYS-PUB-2013-012 (2013), http://cds.cern.ch/record/1604506. 